\title{
EFFECT OF ENERGY BEVERAGES ON THE ENAMEL HARDNESS AND SHEAR BOND STRENGTH OF ORTHODONTIC BRACKETS (IN-VITRO STUDY)
}

\author{
Eman M. ElSayed* and Amr Sharawy**
}

\begin{abstract}
The purpose of the present study was to investigate the effect of energy (sports) drinks on enamel hardness and shear bond strength of orthodontic brackets. Seventy maxillary first premolar teeth were divided into three groups. In Group I, the enamel hardness was measured with Vickers knoop hardness tester, after immersing the teeth in the distilled water (control) and energy drink for , one, two and four weeks. In group II, after 2 weeks of storage in the beverage and distilled water, the teeth were bonded with standard metal stainless steel brackets, using Concise composite resin, after which the SBS was immediately measured. Then the coefficient of correlation between hardness and SBS of group II was recorded. In group III, the teeth were stored in the beverage and water with bonded brackets, as in group I. The SBS was then measured. The 2 weeks and 4 weeks beverage groups showed the significantly lowest enamel hardness values with no difference between them. A positive correlation $(r=0.89)$ was found between enamel hardness and SBS. The 4 weeks beverage storage showed the lowest SBS followed by 2 weeks storage, but the values were clinically acceptable. Thus, it was concluded that energy drinks significantly reduced enamel hardness and SBS, and this reduction was proportional to period of storage.
\end{abstract}

\section{INTRODUCTION}

It was clinically observed that some patients coming with frequent failures of bonded brackets were acidic or soft drinks' consumers. Thus, those drinks could have an adverse effect on enamel surface or adhesive. Many researches studied the effect of chemicals on enamel surface. The effect of bleaching agents on enamel micro hardness was studied and it was found that directly after bleaching $51 \%$ of the treatments showed micro hardness reduction, while $49 \%$ did not yield any reduction (Attin et al., 2009). Several home bleaching agents procedures were studied on their effect on enamel micro hardness. The hardness was measured by a knoop diamond at load of $1 \mathrm{~N}$, applied 30 seconds. The highest reduction on enamel hardness was that

* Lecturer at the Orthodontic department, Faculty of Oral and Dental medicine, Cairo University.

** Assistant professor at the Dental Biomaterials department, Faculty of Oral and Dental Medicine, Cairo University. 
manifested by the agent containing sodium chlorite with citric acid (Zantner et al., 2007). They claimed that artificial saliva worked as re-mineralization solution, and its effect depending on the storage time. Soft drinks were also found to reduce enamel hardness, with no difference between the labial and palatal surfaces. The frequency of intake was not decisive on enamel micro-hardness reductions (Van Eygen et al., 2005), while other studies found it proportional. The beverage effects on etched tooth enamel in simulated oral environment was studied at an interval of 3 times/day for 5 days. Soft drinks caused demineralization, but found that artificial saliva, did not overcome it (Dincer et al., 2002).

Many factors could affect the bond strength of orthodontic brackets (Yamamoto et al., 2006). Those factors could include the bond strength of adhesive, surface topography of enamel, operator technique, bracket system used and masticatory forces in various regions of the oral cavity. Chemicals as antioxidants and chlorhexidine were studied regarding their effect on shear bond strength of brackets (Bulut et al., 2006; Cacciafesta et al., 2006). Those agents significantly lowered the bond strength of brackets. Several studies investigated the effect of soft drinks on enamel surface (Asher et al., 1987; Dincer et al., 2002; Gedalia et al., 1991; Grando et al., 1996; Grenby et al., 1989; Grenby, 1990; Grobler et al., 1990; Ireland et al., 1995; Mistery et al., 1993; Smith and Shaw, 1987; Smith, 1989; Steffen, 1996; Van Eygen, 2005). But to what extent could those drinks affect the bond strength of brackets was not studied. The use of sports beverages had been widely used those days. Thus, the objective of the present study was to identify the effect of the sports (energy) beverage on the enamel micro hardness and shear bond strength of orthodontic brackets. Whether a correlation existed between the enamel micro hardness and shear bond strength of brackets was also investigated.

\section{MATERIAL AND METHODS}

\section{Material}

\section{Teeth:}

Seventy maxillary first premolar teeth were included in the study. They were extracted from patients (ages $=17-24$ years old) seeking orthodontic treatment. The teeth were free from caries, fractures or white spots. They were cleaned from blood and debris, and immediately stored in saline, at $25^{\circ} \mathrm{C}$, until they were used for experiment.

\section{The energy (sports) drink:}

Tonino Lamborghini energy drink (HD world enterprises L.L.C.) was used as the experimental beverage. It contained; carbonated water, sugar, acids(citric acid and sodium citrate), taurine, caffeine, artificial flavours, D-glucuronolactone, inositol, preservatives, colors (caramel and riboflavin), and vitamins (niacin, panthotonic acid, B6, B12).

\section{Methods}

The method was divided into three parts. The first (Group I) was to investigate the effect of the beverage on enamel hardness, under different storage times. The second part (Group II) of the experiment was to examine the correlation between enamel hardness and shear bond strength under the effect of the drink. In Group III, the effect of the sports beverage on the shear bond strength of brackets was investigated.

Group I included thirty teeth. They were further subdivided into six equal subgroups ( 3 control $(a, b, c)$ and 3 experimental $(\mathrm{d}, \mathrm{e}, \mathrm{f})$, each stored at three time intervals; one week, two weeks and four weeks). The teeth in the control subgroups were stored in distilled water at $37^{\circ} \mathrm{C}$. While the experimental group were stored in the Tonino Lamborghini energy drink at $37^{\circ} \mathrm{C}$. At the end of storage, the enamel hardness was measured. 
Group II included ten teeth; 5 control and 5 in the sports beverage. Both subgroups were left for two weeks, after which all the teeth were bonded with the brackets and the shear bond strength was immediately measured (Immediate group).

In Group III, the thirty teeth were bonded with standard edge wise stainless steel premolar brackets. They were subdivided into six subgroups ( 3 control and 3 experimental) and stored in distilled water and beverage at time intervals; one, two and four weeks. The shear bond strength was then measured.

\section{1) Bonding technique:}

Standard stainless steel maxillary premolar brackets (Unitek), with 0.022 inch slot were used. The teeth were cleaned with rotating brush and fine pumice and dried with oil free air syringe. $37 \%$ of phorphoric acid was used for etching for 30 seconds. The teeth were bonded with Concise (3M Unitek, Monrovia, Calif.), a chemically cured bis-GMA (bisphenol glycidyl methacrylate) composite resin. The resin was applied according to manufacturer's instructions at $25^{\circ} \mathrm{C}$, by the same operator at the middle surface of the buccal of the teeth. The residual resin was removed with a scaler.

\section{2) Measurement of $\mathrm{pH}$ :}

The $\mathrm{pH}$ of the tested solution was obtained by using a $\mathrm{pH}$ meter which had been calibrated according to the manufacturer's instructions. The beverage was stored in airtight containers at $37^{\circ} \mathrm{C}$ in the incubator (PS.3A, Advanced Technology, Egypt) prior to use. Fifty $\mathrm{ml}$ of the beverage was placed in a beaker, then the $\mathrm{pH}$ meter inserted and the reading recorded.

\section{3) Surface hardness test:}

After the storage interval, the teeth were taken out of the solutions. The surface microhardness was measured using digital display Vickers microhardness tester (Model HVS-50, Laizhou
Huayin Testing Instrument Co., Ltd. China ) with a Vickers diamond indenter and a $20 \mathrm{X}$ objective lens. A load of 200 gram was applied to the surface of the specimens for $15 \mathrm{sec}$. Three indentations were equally placed over a circle and not closer than 1 $\mathrm{mm}$ to the adjacent indentations or to the margin of the specimens were made on the surface of each specimen. The length of the indentations diagonal was measured by built in scaled microscope.

Vickers micro-hardness was obtained using the following equation: $\mathbf{V H N}=\mathbf{1 . 8 5 4} \mathbf{L} / \mathbf{d}^{2}$

Where; VHN: Vickers hardness number in $\mathrm{Kg} / \mathrm{m}$, L: Load in $\mathrm{Kg}$, d: Average Length of the diagonals in $\mathrm{mm}$.

\section{4) Shear bond strength test:}

A circular interface shear test was used to evaluate the bond strength. All samples (acrylic embedded tooth with its own bonded bracket) were individually mounted onto a computer controlled materials testing machine (Model LRXplus; Lloyd Instruments Ltd., Fareham, UK). The data were recorded using computer software (Nexygen-MT-4.6; Lloyd Instruments). Sample was secured to the lower fixed compartment of testing machine by tightening screws. Shearing test was done by compressive mode of load applied at enamel-resin interface using a mono-beveled chisel shaped metallic rod attached to the upper movable compartment of testing machine traveling at crosshead speed of $0.5 \mathrm{~mm} / \mathrm{min}$. The load required to debonding was recorded in Newton.

\section{Shear bond strength calculation:}

The load at failure was divided by interfacial bonding area to express the bond strength in MPa:

$$
\tau=\mathbf{P} / \pi \mathbf{r}^{2}
$$

Where; $\boldsymbol{\tau}$ : shear bond strength (MPa), P: load at failure $(\mathrm{N}), \boldsymbol{\pi}: 3.14$, $\mathbf{r}$ : radius of disc $(\mathrm{mm})$ 


\section{5) Statistical analysis}

Regression model using Two-way Analysis of Variance (ANOVA) was used in testing significance for the effect of solution, and storage time on shear bond strength of brackets. One-way ANOVA was used for comparison between hardness values. Tukey's post-hoc test was used for pair-wise comparison between the means when ANOVA test is significant. The significance level was set at $P \leq$ 0.05 . Statistical analysis was performed with SPSS 16.0 ${ }^{1 \circledast}$ (Statistical Package for Scientific Studies) for Windows. Pearson's correlation coefficient was used to determine significant correlation between shear bond strength and hardness.

\section{RESULTS}

Data were presented as mean and standard deviation (SD) values.

\section{Vickers hardness test}

Regarding the effect of the energy drink on enamel micro hardness, there was no statistically significant difference between mean hardness values of distilled water and 1 week beverage, both showed the statistically significantly highest mean hardness values. There was no statistically significant difference between mean hardness values of 2 weeks beverage and 4 weeks beverage. (Fig. 1)

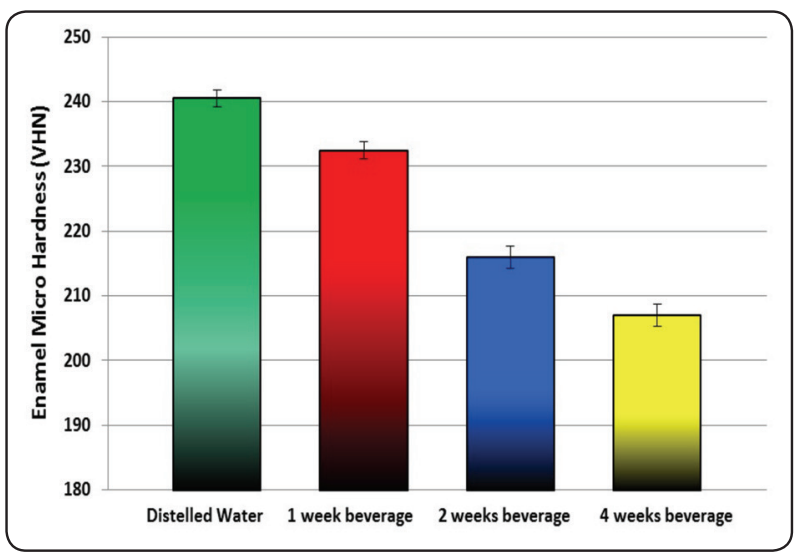

Fig. (1) Bar chart representing means and SD values for comparison between hardness values.

\section{Effect of beverage on shear bond strength (Immediate ):}

The shear bond strength of brackets bonded after two weeks storage in the beverage, showed significantly lower values than in distilled water group. (p $\leq 0.001$ ). (Fig. 2).

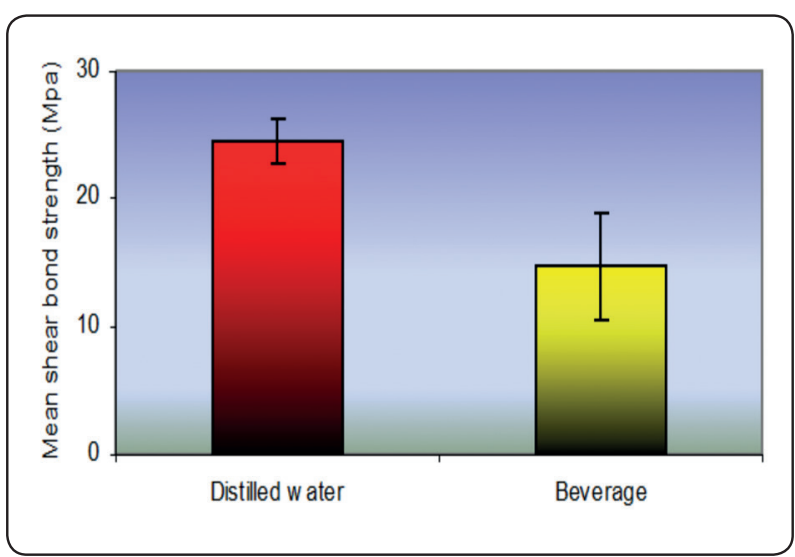

Fig. (2) Bar chart representing means and SD values for comparison between shear bond strength of the two solutions.

\section{Correlation between shear bond strength (SBS) and enamel hardness:}

There was a statistically significant positive (direct) correlation between enamel micro hardness stored in beverage for two weeks, and shear bond strength of brackets. Thus, decrease in enamel hardness would lead to reduced shear bond strength (Fig 3).

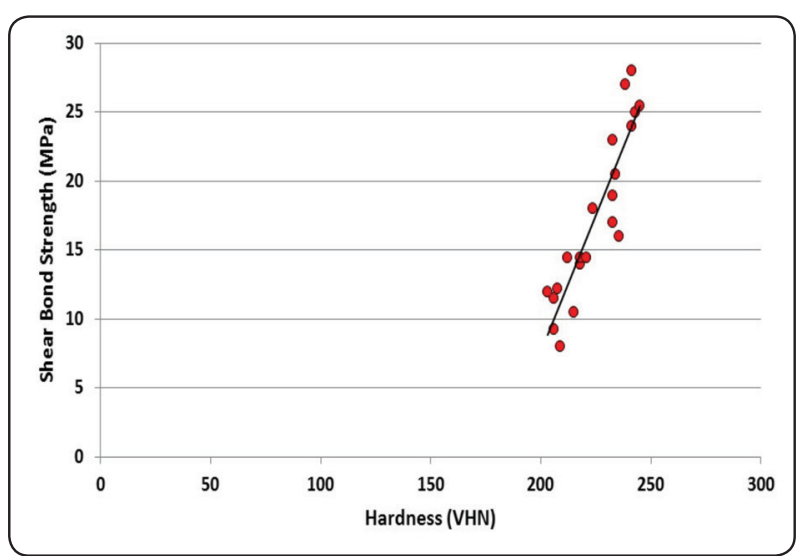

Fig. (3) Scatter diagram showing positive correlation between shear bond strength and enamel hardness.

\footnotetext{
${ }^{\circledR}$ SPSS, Inc., Chicago, IL, USA.
} 


\section{Effect of the storage time on the shear bond strength}

No statistically significant difference was found between shear bond strength of brackets stored in all the times in distilled water (one, two, and four weeks), and the one week storage in the beverage. The lowest statistically significant mean shear bond strength $(\mathrm{p} \leq 0.05)$, was found with the four weeks storage in the beverage followed by the two weeks storage period.(Fig. 4)

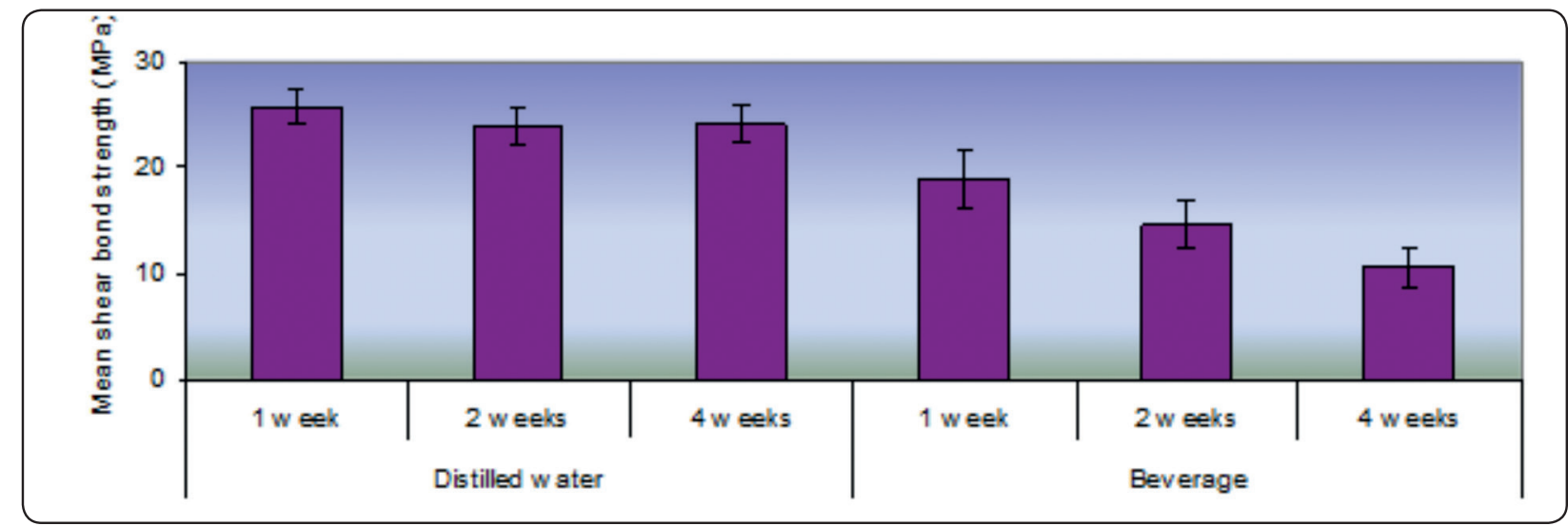

Fig. (4) Bar chart representing means and SD values for comparison between shear bond strength under different storage times.

\section{DISCUSSION}

The teeth included in the study were from patients aging (17-25 years old), to exclude the effect of age changes on enamel structure. The test was performed at $37^{\circ} \mathrm{C}$ to simulate oral cavity temperature. The effect of the energy drink on enamel hardness was studied, and hence how much this would affect the teeth of patients who would seek orthodontic treatment. The beverage was found to significantly reduce the enamel micro-hardness. This agreed with studies done by (Van Eygene et al., 2005) on soft drinks, but they investigated that reduced frequency of intake of the drinks was not proportional with reduction in tissue loss. In this study the increase of immersion of teeth in the beverage significantly reduced enamel micro hardness from 232.5+1.9 $\mathrm{MPa}$ (1 week),to 216+7.4MPa (2 weeks), to $207.7+4.8 \mathrm{MPa}$ (4 weeks). This result was also claimed by (Lussi et al., 2000). Several studies tested the enamel dissolution by soft drinks, by scanning electron microscope. It was proposed that the longer immersions of human and bovine teeth, in cola-type drinks, the more was the dissolution of enamel prism cores and interprismatic areas (Meurman et al., in 1991 and 1996). The severity of decalcification areas was increased with more exposure to cola and fruit juices
(Grando et al., 1996; Dincer et al., 2002). The contradiction between the studies could have been due to the difference in the way of immersion of teeth in the drinks. Those that found more enamel loss, or decrease in micro hardness, with the increase in immersion times, the immersion was continuously. While those that the frequency did not affect the enamel loss, the immersion of teeth was in intervals with artificial saliva. Thus, they claimed that artificial saliva caused enamel re-mineralization. But other studies found that saliva did not overcome the demineralization of soft drinks.

As we found an effect of the beverage on enamel hardness, we also examined how much would this change in enamel affect the bonding of orthodontic brackets. Patients come to our orthodontic clinics already consuming those soft or energy drinks. Thus, they already had a change in enamel microhardness. Consequently a sample of teeth was left for two weeks period (medium beverage intake), after which brackets were bonded, then the shear bond strength was immediately measured. A positive correlation ( $\mathrm{r}=0.89)$ was recorded between the enamel micro-hardness and shear bond strength of brackets. One study was reported that found a posi- 
tive correlation between the enamel hardness and in-vitro shear bond strength (Grenby, 1990). Thus, certain precautions should be taken when bonding brackets to patients who are already energy drinks' consumers. This could include, using brackets of strong retentive bases (Lopez, 1980; Wang et al. 2004), or adhesives with high bond strength. Wang et al. in 2004 claimed that the shear bond strength of bracket bases in order from highest to lowest were; Tomy, Dentaurum, Unitek, Leone, TP Orthodontics, and lastly Ormco brackets. The brackets used in the study were Unitek, with mesh base, where its base bond strength was considered strong retention.

The second part of the experiment was to test the effect of the energy drinks on shear bond strength of brackets. No data were available to identify if that effect of the beverages on shear bond strength was investigated. In our study, the shear bond strength was significantly affected by the beverage. The bond strength decreased from $19+2.82$ to $10.63+1.88$ during one to four weeks storage period, respectively. Previous researches studied the effect of other chemicals as chlorhexidine mouth was (Cacciafesta et al. 2006) and bleaching agents (Attin et al., 2009; Bulut et al., 2006; Zantner, 2007) on shear bond strength of brackets. Bulut et al., studied the effect of one week immersion of teeth in the bleaching agent, $10 \%$ carbamide peroxide $(\mathrm{CP})$. In their study they used the same adhesive (Concise,3M Unitek), as in this study. The shear bond strength values decreased from 20.6+2.9 MPa (control) to 14.2+2.4 MPa (one week immersion in $\mathrm{CP}$ ). The values of bond strength of the one weeks immersion in $\mathrm{CP}$ were similar to the two weeks immersion in energy drink in the present study. Thus, CP had a more reducing effect on SBS, than energy drink of the same period of immersion. Many studies compared the shear bond strength of different adhesives. Resin-modified glass ionomer adhesive $(9.56 \pm 1.85 \mathrm{MPa})$, had significantly less SBS than light cured conventional resin adhesive (18.46 $\pm 2.9 \mathrm{MPa})$ (Summers at al., 2004). In other studies Transbond XT (3M Unitek) etched with 37\% phosphoric acid, yielded the highest SBS $(19 \pm 6.7$
Mpa) than self-etching primers and adhesives (ranging from $10.1 \pm 4.7 \mathrm{MPa}$ ). While Transbond plus with Transbond XT (3M Unitek) showed shear bond strength of (16.9 \pm 7.3 MPa) (Vilchis et al., 2009). Previous investigations claimed that the range from 2.8 to $10 \mathrm{MPa}$ of shear bond strength was clinically acceptable. They stated that although enamel can often withstand much greater forces during debonding, the prudent clinician should avoid using bracket conditioner adhesive combinations that can result in bond strengths greater than 13.5 MPa. This was claimed, to avoid enamel fractures during de-bonding (Lopez, 1980 and Reynolds, 1975). Thus, in this study despite the changes in enamel hardness, the bond strength values were even above the clinically acceptable values. Consequently, the adhesive also played a major role in the success of bonding. As we have wide variations of adhesives and bracket base SBS, so we can select the most suitable combinations for each patient, individually.

\section{CONCLUSIONS}

- Enamel hardness was significantly reduced by the energy soft drink, and the more immersion in the beverage the more was the reduction.

- Enamel surfaces affected by the drinks would affect the SBS of the brackets.

- The energy drink significantly affected the SBS, and was proportional to the period of immersion.

- The combination of bracket-adhesive systems would greatly influence the success of orthodontic bonding, in patients who are energy drink consumers. The combination used in this study for those patients, had a clinically acceptable SBS.

\section{REFERENCES}

- Asher C, Read MJF: Early enamel erosion in children associated with the excessive consumption of citric acid. Br Dent J 162:384-387. 1987.

- Attin T, Schmidlin PR, Wegehaupt F, Wiegand A: Influence of study design on the impact of bleaching agents on dental enamel micro hardness. A review. Dental materials 25:143-157. 2009. 
- $\quad$ Bhad WA, Hazaney PV: Scanning electron microscopic study and shear bond strength measurement with $5 \%$ and $37 \%$ phosphoric acid. Am J Orthod Dentofacial Orthop 108:410-414. 1995.

- $\quad$ Bishara SE, Ajlouni R, Laffoon J and Warren J : Effects of modifying the adhesive composition on the bond strength of orthodontic brackets. Angle Orthod 72: 464-467.2002.

- $\quad$ Bulut H, Turkun M, Kaya AD : Effect of an antioxidizing agent on the shear bond strength of brackets bonded to bleached human enamel. Am J Orthod Dentofacial Orthop 129(2):266-272. 2006.

- Cacciafesta V, Sfondrini MF, Stifanelli P, Scribante A, Klersy C: Effect of chlorhexidine application on shear bond strength of brackets bonded with a resin-modified glass ionomer. Am J Orthod Dentofacial Orthop 129 (2):273-276. 2006.

- Dincer B, Hazar S, Sen BH: Scanning electron microscope study of the effects of soft drinks on etched and sealed enamel. Am J Orthod Dentofacial Orthop 122:135-41. 2002.

- Eccles JD, Jenkins WD: Dental erosion and diet. J Dent 2:153-7. 1974.

- Eccles JD: Dental erosion of nonindustrial origin: a clinical survey and classification J Prosthet Dent, 42:64953. 1979.

- Gedalia I, Ionat-Bendat S, Ben-Mosheh S, Shapira L: Tooth enamel softening with a cola type drink and rehardening with hard cheese or simulated saliva in situ. J Oral Rehabil 18:501-506. 1991.

- Grando LJ, Tames DR, Cardoso AC, Galiban NH: In vitro study of enamel erosion caused by soft drinks and lemon juice in deciduous teeth analysed by stereomicroscopy and scanning electron microscopy. Caries Res 30:373-378. 1996.

- Grenby TH, Phillips A, Desai T, Mistry M: Laboratory studies of the dental properties of soft drinks. Br J Nutr 62:451-64. 1989.

- Grenby TH: In vitro experiments on effect of soft drinks on dental enamel. Oral prophylaxes. 12:103-113. 1990.

- Grobler SR, Senekai PJC, Laubscher JA: In vitro demineralization of enamel by orange juice, apple juice, Pepsi-Cola and diet cola. Clin Prev Dent 12:5-9. 1990.

- Ireland AJ, McGuinness N, Sherriff M: An investigation into the ability of soft drinks to adhere to enamel. Caries Res 29:470-476. 1995.

- $\quad$ Lussi A, Kohler N, Zero D, Schaffner M, Megert B: A comparison of the erosive potential of different beverages in primary and permanent teeth using an in vitro model. Eur J Oral Sci 108(2):110-114. 2000.

- Lopez JI: Retentive shear strengths of various bonding attachment bases. Am J Orthod 77:669-678. 1980.
- Lou L, Heo G, Nelson AE, Alsagheer A, Carey JP, Major PW: Chemical composition of enamel surface as a predictor of in-vitro shear bond strength. Am J Orthod Dentofacial Orthop 136(5):683-688. 2009.

- Mannerberg F: Saliva factors in cases of erosion. Odontol Rev 14:156-166. 1963.

- Meurman JH, Drysdale T, Frank RM: Experimental erosion of dentin. Eur J Oral Sci 99(6):457-462. 1991.

- Meurman JH, ten Gate JM: Pathogenesis and modifying factors of dental erosion. Eur J Oral Sci 104(2):199-206.1996.

- Mistry M, Grenby TH: Erosion by soft drinks of rat molar teeth assessed by digital image analysis. Caries Res 27:2125.1993

- O'Reilly M, Featherstone J: Demineralization and remineralization around orthodontic appliances: an in vivo study. Am J Orthod Dentofacial Orthop 92:33-40. 1987.

- Retief DH : Failure of the dental adhesive-etched enamel interface. J Oral Rehabil 1:265. 1974.

- Reynolds IR:.A review of direct orthodontic bonding. Br J Orthod 2:171-178. 1975.

- $\quad$ Smith BGN, Knight JK: An index for measuring wear of teeth. Br Dent J 156:435-438. 1984.

- Smith AJ, Shaw L: Baby fruit juices and tooth erosion. Br Dent J 162:65-67. 1987.

- Smith BGN: Toothwear: aetiology and diagnosis. Dent Update 16:204-212. 1989.

- Steffen MJ: The effects of soft drinks on etched and sealed enamel. Angle Orthod 66:449-456. 1996.

- Summers A, Kao E, Gilmore J, Gunel E, Ngan P: Comparison of bond strength between a conventional resin adhesive and a resin-modified glass ionomer adhesive: An in vitro and in vivo study. Am J Orthod Dentofacial Orthop 126 (2):200-206. 2004.

- Van Eygen I, Vande Vannet B, Wehrbein H. Influence of soft drink with low $\mathrm{pH}$ on enamel surfaces: An in vitro study: Am J Orthod Dentofacial Orthop 128:372-377.2005.

- Vilchis RIS, Yamamoto S, Kitai N, Yamamoto K. Shear bond strength of orthodontic brackets bonded with selfetching adhesives: Am J Orthod Dentofacial Orthop 136(3):425-430. 2009.

- Wang WN, Li CH, Chou TH, Wang DDH, Lin LH, Lin CT: Bond strength of various bracket base designs. Am J Orthod Dentofacial Orthop 125(1) 65-70. 2004.

- Yamamoto A, Yoshida T, Tsubota K, Takamizawa T, Kuvokawa H, Miyazaki M. Orthodontic bracket bonding: Enamel bond strength vs time: Am J Orthod Dentofacial Orthop 130 (4):435.e1-435.e6. 2006.

- Zantner C, Beheim-Schwazbach N, Neumann K, Keilbassa AM. Surface micro-hardness of enamel after different home bleaching procedures: Dental materials 23:243-250. 2007. 